\title{
ONE-SIDED BOUNDARY BEHAVIOR FOR CERTAIN HARMONIC FUNCTIONS
}

\author{
T. K. BOEHME AND MAX L. WEISS ${ }^{1}$
}

\begin{abstract}
Amstract. Some results concerning the maximal ideal space of $H^{\infty}$ of the disk are applied to harmonic functions. The methods yield a Lindelöf type theorem for harmonic functions and extend to bounded harmonic functions a criterion of Tanaka which is necessary and sufficient in order that the boundary value function be one-sided approximately continuous.
\end{abstract}

1. Introduction. We are concerned in this paper with connections between the one-sided behavior of an $L^{\infty}$ function at a point of the unit circle, $C=\{z:|z|=1\}$, and the boundary behavior of the harmonic extension of the function into the unit disc, $D=\{z:|z|<1\}$. Our techniques consist mainly of combining certain concrete estimates for harmonic measures with some facts about the Banach algebra, $H^{\infty}$, of bounded analytic functions on $D$. We assume for this latter area that the reader is familiar with the contents of Chapter 10 of Hoffman's book, [3], and with [4] and [5].

The main focal point is the introduction of a class of homomorphisms in the maximal ideal space of $H^{\infty}$ which we call the "barely tangential homomorphisms." These homomorphisms play a role for the one-sided boundary behavior of $L^{\infty}$ functions similar to that played by the radial homomorphisms for two-sided behavior in [1].

$\$ 2$ is mainly devoted to an intrinsic study of the barely tangential homomorphisms. In $\$ 3$ we obtain a theorem (Theorem 3.1) characterizing one-sided approximate continuity from above of an $L^{\infty}$ function in terms of the behavior of the function on the supports of the representing measures of upper barely tangential homomorphisms. Subsequently, we easily obtain a result of Tanaka [6, Theorem 5] characterizing one-sided approximate continuity. As a final result we prove a "Lindelöf-type theorem" for $L^{\infty}$ functions.

2. Barely tangential homomorphisms. We first recall that the collection, $H^{\infty}$, of bounded analytic functions on $D$ forms a function algebra with pointwise operations and the supremum norm. Its maximal ideal space, $D$, is a compactification of $D,[3]$. Every homomorphism in $D$ can be approached by a universal net in $D$ or by one

Received by the editors February 19, 1970.

AMS 1969 subject classifications. Primary 3120.

Key words and phrases. Boundary behavior, harmonic functions.

${ }^{1}$ Both authors were supported in part by NSF Grant GP 13284. 
in any dense subset of $D$. One can also represent $H^{\infty}$ as a subalgebra of the Banach algebra, $L^{\infty}$, of all bounded measurable functions on $C$. We rely on the references for a complete description of these connections. We remark here that for any $L^{\infty}$ function $f$ on $C$ we will continue to denote as " $f$ " any of the standard representations of $f$ on $D$, $D$, or $C$.

For simplicity we restrict our attention to the fiber, $D_{1}$, above 1 , i.e., those homomorphisms which are approached by nets tending to 1 . From here on out we will simply assume phrases such as, "at 1." The collection, $\mathcal{S}$, of such homomorphisms which are approached within a Stolz angle are called the Stolz homomorphisms. In [5] the $w^{*}$ closure, $\mathfrak{S}^{-}=\mathfrak{L}$, of the Stolz homomorphisms is called the Lindelöf homomorphisms.

Definition 2.1. Let $\delta$ and $\&$ be the Stolz and Lindelöf homomorphisms, respectively. Then, the collection, $B=\mathfrak{L}-\mathcal{S}$, is called the barely tangential homomorphisms. Those points, $\mathbb{B}^{+}\left[\mathbb{B}^{-}\right]$, of $\mathbb{B}$ which are approached by nets tending to 1 tangentially from above [below] are termed the upper [lower] barely tangential homomorphisms.

Our first result shows a relationship between $B$ and $\mathscr{L}$ which we shall use once in $\S 3$. We recall that for any function algebra, $A$, on a compact Hausdorff space $X$, each point $h \in X$ has a representing measure, $\mu_{h}$, spread on the Silov boundary of $A$.

Lemma 2.2. Let $A$ denote the restriction algebra of $H^{\infty}$ to the Lindelöf homomorphisms, \&. Then, the Silov boundary of $A$ is contained in the barely tangential homomorphisms, B. If $h$ is a Stolz homomorphism, then for any representing measure $\mu_{h}$ we have $\mu_{h}\left(B^{+}\right)>0$ and $\mu_{h}\left(B^{-}\right)>0$. In particular, if $f \in H^{\infty}$, then $f$ has radial limit $\alpha$ if and only if $f$ is constantly $\alpha$ on $B^{+}$(or on $\left.B^{-}\right)$.

Proof. We show that no Stolz homomorphism is contained in the Silov boundary. Let $h \in \delta$. Since by $[5, \S 3] \delta$ is open in $D_{1}$ and since $B$ is compact there is a neighborhood $N$ of $h$ such that $N \subset S$ and $N \cap \AA=\varnothing$. Now, suppose $f \in H^{\infty}$ and $f$ peaks on $N$, i.e., $\left|f\left(h_{1}\right)\right|=\|f\|_{\mathscr{L}}$ for some $h_{1} \in N$. Choose some Gleason part, $P$, of $D$ which contains such a point in $N$. Using the results of [5, (especially $\S 6)]$ we see that $P \subset \delta$ and $P-\cap \beta \neq \varnothing$. Since the restriction of $f$ to $P$ is analytic and achieves its maximum modulus on $P$ it is constant. Thus $f$ peaks at some point of $P-\cap \Re$. Consequently, we have shown that $f$ peaks outside $N$. Therefore, $h$ cannot be in the Šilov boundary and the latter is contained in $B$.

Next, let $h \in \mathcal{S}$ and let $\mu_{h}$ be a representing measure. Let $u$ be the harmonic measure of $C^{+}=\left\{e^{i \theta}: 0 \leqq \theta \leqq \pi\right\}$. Let $v$ be a harmonic con- 
jugate and let $f=\exp (u+i v)$. Then $|f|=e$ on $B^{+},|f|=1$ on $B^{-}$ and $1<|f|<e$ on $\mathscr{L}$. If $\mu_{h}\left(\mathbb{B}^{+}\right)=0$, then we would have $|f(h)| \leqq 1$ which is impossible. Thus $\mu_{h}\left(\mathbb{B}^{+}\right)>0$, and similarly $\mu_{h}\left(B^{-}\right)>0$.

Finally, suppose $f \in H^{\infty}$. If $f$ has radial limit $\alpha$, then $f$ has Stolz limit $\alpha$ and thus $f=\alpha$ on $S$. Consequently, by continuity $f=\alpha$ on $\mathbb{B}^{+}$ $\left(\right.$ or $\left.\mathbb{B}^{-}\right)$. Conversely, suppose $f=\alpha$ on $\mathbb{B}^{+}\left(\right.$or $\left.\mathbb{B}^{-}\right)$. Let $\mu_{h}$ be a Jensen measure for $h \in \mathcal{S}$. Then,

$$
\log |f(h)-\alpha| \leqq \int_{\mathbb{B}^{+} \cup B^{-}} \log |f-\alpha| d \mu_{h} .
$$

Since $\mu_{h}\left(B^{+}\right)>0$ and $f-\alpha=0$ on $\mathbb{B}^{+}$, the integral above equals $-\infty$ and so $f(h)=\alpha$. Thus, $f=\alpha$ on $S$ and $a$ fortiorif $f$ tends to $\alpha$ radially.

In order to avoid exactly similar cases we now concentrate entirely on the upper barely tangential homomorphisms.

We next define a cluster set which will allow us to state more concretely Theorem 3.3 in \$3. Each Stolz angle approach can be written as $\theta=c(1-r)$ and as $c$ increases the approach tends more and more toward an upper tangential approach. Given $d \leqq c$ we denote by $S_{d, c}$ the collection of all homomorphisms in $D_{1}$ which are approached by nets corresponding to all Stolz angle approaches, $a$, such that $d \leqq a \leqq c$. Given $f \in L^{\infty}$ define $S_{d, c}(f, 1)$ as the collection of all cluster values of $f\left(r e^{i \theta}\right)$ as $r e^{i \theta} \rightarrow 1$ and $d \leqq \lim$ inf $\theta /(1-r) \leqq \lim \sup \theta /(1-r) \leqq c$. It is not hard to see that we then have $S_{d, c}(f, 1)=f\left[\delta_{d, c}\right]$. Now, define $B^{+}(f, 1)$, the upper barely tangential cluster set of $f$ at 1 by

$$
B^{+}(f, 1)=\bigcap_{d \geq 0}\left[\bigcup_{c \geq 0} S_{d, c}(f, 1)\right]^{-} .
$$

Lemma 2.3. Let $f \in L^{\infty}$. Then, $B^{+}(f, 1)=f\left[B^{+}\right]$.

Proof. Suppose $h_{0} \in \mathbb{B}^{+}$. Then, there is a net $h_{\alpha} \in \mathcal{S}$ such that $h_{\alpha} \rightarrow h_{0}$. Clearly, for each $d \geqq 0, h_{\alpha}$ is eventually in $U_{c \geq 0} \delta_{d, c}$. Thus, $h_{0} \in \bigcap_{d \geq 0}\left[U_{c \geq 0} S_{d, c}\right]^{-}$. On the other hand suppose $h_{0} \in \bigcap_{d \geq 0}\left[U_{c \geq 0} \delta_{d, c}\right]^{-}$. Then, for each $d \geqq 0, h_{0} \in\left[U_{c \geq 0} S_{d, c}\right]-$. Since $\left[U_{c \geq 0} S_{d, c}\right]--S=B^{+}$for each $d, h_{0} \in B^{+}$. Therefore,

$$
B^{+}=\bigcap_{d \geq 0}\left[\bigcup_{c \geq 0} S_{d, c}\right]^{-}
$$

Since the intersection is over a nested system of compact sets and since $f$ is continuous on $D$ we have

$$
f\left[B^{+}\right]=\bigcap_{d \geq 0}\left[\bigcup_{c \geq 0} f\left[s_{d, c}\right]\right]^{-}=B^{+}(f, 1) .
$$


Given a measurable subset, $M$, of the circle, $C$, we let $u_{M}$ denote the harmonic measure of $C$,

$$
u_{M}\left(r e^{i \theta}\right)=\frac{1}{2 \pi} \int_{-\pi}^{\pi} \chi_{M}\left(e^{i t}\right) \frac{1-r^{2}}{1-2 r \cos (\theta-t)+r^{2}} d t
$$

where $\chi_{M}$ is the characteristic function of $M$. If $M$ is a subset of the upper portion of the unit circle, $C^{+}=\left\{e^{i \theta}: 0 \leqq \theta \leqq \pi\right\}$, we let

$$
d(M)=\liminf _{\theta \rightarrow 0^{+}} \frac{1}{\theta} \int_{0}^{\theta} \chi_{M}\left(e^{i t}\right) d t, \quad D(M)=\lim _{\theta \rightarrow 0^{+}} \sup \frac{1}{\theta} \int_{0}^{\theta} \chi_{M}\left(e^{i t}\right) d t
$$

denote the lower and upper densities of $M$ at 1 from above.

Our basic results rely heavily on the following estimates for harmonic measures.

Lemma 2.4. Let $M$ be a measurable subset of $C^{+}$. If $d(M)=D(M)$, then for every $h \in B^{+}, u_{M}(h)=d(M)$.

Lemma 2.5. Let $M$ be a measurable subset of $C^{+}$. Then, there exists an $h \in{B^{+}}^{+}$with

$$
u_{M}(h) \geqq \frac{2}{\pi} \tan ^{-1} \frac{D(M)}{2 \sqrt{ }(1-D(M))} .
$$

Proof OF 2.4. Let $\epsilon>0$ and choose $0<\theta_{0}<\pi / 2$ so that, for $0 \leqq \theta \leqq \theta_{0}$,

$$
d(M)-\epsilon<\frac{1}{\theta} \int_{0}^{\theta} \chi_{M}\left(e^{i t}\right) d t \leqq d(M)+\epsilon .
$$

Since the harmonic measure of $M \cap\left[\theta_{0}, \pi\right]$ tends to zero as $z \rightarrow 1$ we have, as $z \rightarrow 1$,

$$
u_{M}\left(r e^{i \theta}\right)=o(1)+\frac{1}{2 \pi} \int_{0}^{\theta_{0}} P_{r}(\theta-t) \chi_{M}\left(e^{i t}\right) d t
$$

where $P_{r}(t)$ is the Poisson kernel. Integrating by parts we have

$$
\begin{aligned}
\frac{1}{2 \pi} \int_{0}^{\theta_{0}} P_{r}(\theta-t) \chi_{M}\left(e^{i t}\right) d t= & \frac{P_{r}\left(\theta-\theta_{0}\right)}{2 \pi} \int_{0}^{\theta_{0}} \chi_{M}\left(e^{i s}\right) d s \\
& +\frac{1}{2 \pi} \int_{0}^{\theta_{0}} P_{r}^{\prime}(\theta-t) \int_{0}^{t} \chi_{M}\left(e^{i s}\right) d s d t .
\end{aligned}
$$

Thus, as $z \rightarrow 1$,

$$
u_{M}\left(r e^{i \theta}\right)=o(1)+\frac{1}{2 \pi} \int_{0}^{\theta_{0}} P_{r}^{\prime}(\theta-t) \int_{0}^{t} \chi_{M}\left(e^{i s}\right) d s d t .
$$


For $0 \leqq t \leqq \theta<\pi / 2, P_{r}^{\prime}(\theta-t) \leqq 0$ so $(d(M)+\epsilon) t P_{r}^{\prime}(\theta-t) \leqq P_{r}^{\prime}(\theta-t) \int_{0}^{t} \chi_{M}\left(e^{i \theta}\right) d s \leqq(d(M)-\epsilon) t P_{r}^{\prime}(\theta-t) ;$ while, for $\theta \leqq t \leqq \theta_{0}, P_{r}^{\prime}(\theta-t) \geqq 0$ so $(d(M)-\epsilon) t P_{r}^{\prime}(\theta-t) \leqq P_{r}^{\prime}(\theta-t) \int_{0}^{t} \chi_{M}\left(e^{i s}\right) d s \leqq(d(M)+\epsilon) t P_{r}^{\prime}(\theta-t)$.

Therefore,

$$
\begin{aligned}
o(1)+ & \frac{d(M)+\epsilon}{2 \pi} \int_{0}^{\theta} t P_{r}^{\prime}(\theta-t) d t+\frac{d(M)-\epsilon}{2 \pi} \int_{\theta}^{\theta_{0}} t P_{r}^{\prime}(\theta-t) d t \\
& \leqq u_{M}\left(r e^{i \theta}\right) \\
& \leqq o(1)+\frac{d(M)-\epsilon}{2 \pi} \int_{0}^{\theta} t P_{r}^{\prime}(\theta-t) d t+\frac{d(M)+\epsilon}{2 \pi} \int_{\theta}^{\theta_{0}} t P_{r}^{\prime}(\theta-t) d t .
\end{aligned}
$$

Now,

$$
\begin{aligned}
& \int_{0}^{\theta} t P_{r}^{\prime}(\theta-t) d t=\frac{-(1+r) \theta}{1-r}+2 \tan ^{-1}\left\{\frac{1+r}{1-r} \tan \frac{\theta}{2}\right\}, \\
& \int_{0}^{\theta_{0}} t P_{r}^{\prime}(\theta-t) d t=o(1)+\frac{(1+r) \theta}{1-r}+2 \tan ^{-1}\left\{\frac{1+r}{1-r} \tan \frac{\theta_{0}-\theta}{2}\right\},
\end{aligned}
$$

so that if $r e^{i \theta} \rightarrow 1$ in such a way that $\theta /(1-r) \rightarrow c \geqq 0$, we have

$$
\begin{aligned}
(d(M)+\epsilon)[ & {\left[\frac{-c}{\pi}+\frac{\tan ^{-1} c}{\pi}\right]+(d(M)-\epsilon)\left[\frac{c}{\pi}+\frac{1}{2}\right] } \\
& \leqq \\
& \leqq\left(d i m \inf u_{M}\left(r e^{i \theta}\right) \leqq \lim \sup u_{M}\left(r e^{i \theta}\right)\right. \\
& {\left[\frac{-c}{\pi}+\frac{\tan ^{-1} c}{\pi}\right]+(d(M)+\epsilon)\left[\frac{c}{\pi}+\frac{1}{2}\right] . }
\end{aligned}
$$

Since this is true for every $\epsilon>0$, we have as $r e^{i \theta} \rightarrow 1, \theta /(1-r) \rightarrow c \geqq 0$, $d(M)\left[\frac{1}{2}+\frac{\tan ^{-1} c}{\pi}\right]$

$$
\leqq \lim \inf u_{M}\left(r e^{i \theta}\right) \leqq \lim \sup u_{M}\left(r e^{i \theta}\right) \leqq d(M)\left[\frac{1}{2}+\frac{\tan ^{-1} c}{\pi}\right] .
$$

Thus, as $r e^{i \theta} \rightarrow 1, \theta /(1-r) \rightarrow c \geqq 0$, 


$$
\lim u_{M}\left(r e^{i \theta}\right)=d(M)\left[\frac{1}{2}+\frac{\tan ^{-1} c}{\pi}\right] .
$$

Thus, for any homomorphism, $h_{c}$, in $S_{c, c}$ we have

$$
u_{M}\left(h_{c}\right)=d(M)\left[\frac{1}{2}+\frac{\tan ^{-1} c}{\pi}\right] \text {. }
$$

If $h \in B^{+}$, it is the limit of homomorphisms $h_{c}$ for which $c \rightarrow \infty$. Thus, because $u_{M}$ is continuous we have, letting $c \rightarrow \infty, u_{M}(h)=d(M)$, as claimed.

Proof of 2.5. Let $D=D(M)$, let $\epsilon>0$, and pick $\theta_{n} \rightarrow 0$ such that

$$
\frac{1}{2 \theta_{n}} \int_{0}^{2 \theta_{n}} \chi_{M}\left(e^{i t}\right) d t \geqq D-\epsilon .
$$

Then,

$$
\begin{aligned}
u_{M}\left(r e^{i \theta_{n}}\right) & \geqq \int_{0}^{2 \theta_{n}} P_{r}\left(\theta_{n}-t\right) \chi_{M}\left(e^{i t}\right) d t \\
& \geqq 2 \int_{0}^{(D-\epsilon) \theta_{n}} P_{r}\left(\theta_{n}-t\right) d t=2 \int_{(1-D+\epsilon) \theta_{n}}^{\theta_{n}} P_{r}(t) d t \\
& =\frac{2}{\pi} \tan ^{-1}\left\{\frac{\frac{1+r}{1-r}\left(\tan \frac{\theta_{n}}{2}-\tan \frac{(1-D+\epsilon) \theta_{n}}{2}\right)}{1+\left(\frac{1+r}{1-r}\right)^{2} \tan \frac{\theta_{n}}{2} \tan \frac{(1-D+\epsilon) \theta_{n}}{2}}\right\} .
\end{aligned}
$$

Let $r_{n}$ be determined by $\theta_{n}=c\left(1-r_{n}\right)$. Then, the limit as $\theta_{n} \rightarrow 0$ of the numerator of the argument of $\tan ^{-1}$ in the last expression is $c(D-\epsilon)$, while that of the denominator is $1+c^{2}(1-D+\epsilon)$. Hence,

$$
\lim _{\theta_{n} \rightarrow 0} \sup _{M}\left(r_{n} e^{i \theta_{n}}\right) \geqq \frac{2}{\pi} \tan ^{-1}\left\{\frac{c(D-\epsilon)}{1+c^{2}(1-D+\epsilon)}\right\} .
$$

This being true for every $\epsilon>0$, we have

$$
\lim _{\theta_{n} \rightarrow 0} \sup _{M}\left(r_{n} e^{i \theta_{n}}\right) \geqq \frac{2}{\pi} \tan ^{-1}\left\{\frac{c D}{1+c^{2}(1-D)}\right\} .
$$

In particular, if we choose $c=(1-D)^{-1 / 2}$,

$\lim \sup u_{M}\left(r e^{i \theta}\right) \geqq \frac{2}{\pi} \tan ^{-1}\left\{\frac{D}{2 \sqrt{ }(1-D)}\right\}, \quad r e^{i \theta} \rightarrow 1, \quad \theta=c(1-r)$. 
This quantity is therefore a lower bound for $u_{M}(h)$ for some $h \in \delta_{c, c}$. Therefore, by Lemma 2.2 it is also a lower bound for $u_{M}(h)$ for some $h \in ß$. Since $u_{M}=0$ on $B^{-}$, it follows that for some homomorphism $h$ in $B^{+}$,

$$
u_{M}(h) \geqq \frac{2}{\pi} \tan ^{-1}\left\{\frac{D}{2 \sqrt{ }(1-D)}\right\}
$$

as was to be proved.

If $M$ is a measurable subset of $C$, we let $\tilde{M}=\left\{h \in D_{1}: \chi_{M}(h)=1\right\}$. We recall that the range of $f \in L^{\infty}$ on $\tilde{M}$ is precisely the collection of essential cluster values of $f\left(e^{i \theta}\right)$ as $e^{i \theta} \rightarrow 1$ through $M$. We also recall the result from [1] that for each $h \in \mathbb{D}, \mu_{h}(\tilde{M})=u_{M}(h)$. With these preliminaries we may now prove

Corollary 2.6. Let $M$ be a measurable subset of $C^{+}$. Then, $d(M)=1$ if and only if $\mu_{h}(\tilde{M})=1$ for every $h \in B^{+}$.

Proof. If $d(M)=1$, we have from Lemma 2.4 that $u_{M}(h)=1$. From the above remark it is immediate that $\mu_{h}(\tilde{M})=1$. On the other hand suppose $d(M)<1$. Then, $D(\sim M)>0$ and so by Lemma 2.5 there is an $h \in B^{+}$with

$$
u_{\sim M}(h) \geqq \frac{2}{\pi} \tan ^{-1} \frac{D(\sim M)}{2 \sqrt{ }(1-D(\sim M))}>0 .
$$

Since $u_{\sim M}+u_{M}=1, u_{M}(h)<1$ so $\mu_{h}(\tilde{M})<1$ for some $h \in B^{+}$.

3. Applications. It is now an easy matter to obtain several results connecting the one-sided behavior of an $L^{\infty}$ function on $C$ at 1 with its boundary behavior at 1 from inside $D$.

A function $f$ on $C$ is approximately continuous from above at 1 with value $\alpha$ if for every $\epsilon>0$, the density $d\left(\left\{e^{i \theta}:\left|f\left(e^{i \theta}\right)-\alpha\right| \leqq \epsilon, 0 \leqq \theta \leqq \pi\right\}\right)$ equals one. The main theorem upon which the applications are based is

THEOREM 3.1. Let $f \in L^{\infty}$. Then $f$ is approximately continuous from above at 1 with value $\alpha$ if and only if $f$ is identically $\alpha$ on the support of the representing measure of each upper barely tangential homomorphism.

Proof. For each $\epsilon>0$ let $M_{\epsilon}=\left\{e^{i \theta}:\left|f\left(e^{i \theta}\right)-\alpha\right| \leqq \epsilon, 0 \leqq \theta \leqq \pi\right\}$. Then, $f$ is approximately continuous from above at 1 with value $\alpha$ if and only if $d\left(M_{\epsilon}\right)=1$ for every $\epsilon>0$ if and only if, by Corollary 2.6, $\mu_{h}\left(\tilde{M}_{\epsilon}\right)=1$ for every $\epsilon>0$ and every $h \in B^{+}$. This latter statement implies that for each $h \in B^{+}$, the support of $\mu_{h}$ is contained in $\tilde{M}_{\epsilon}$ for 
every $\epsilon$. But on $\tilde{M}_{e},|f(h)-\alpha| \leqq \epsilon$. Thus for each $h \in \mathbb{B}^{+}, f$ is identically $\alpha$ on the support of $\mu_{h}$. On the other hand suppose for each $h \in B^{+}$that $f=\alpha$ on the support of $\mu_{k}$. Then, since $|f-\alpha| \geqq \epsilon$ on $\left(C-M_{\epsilon}\right) \sim$ it must be that the support of $\mu_{h}$ is entirely contained in $\tilde{M}_{\epsilon}$, i.e., $\mu_{h}\left(\tilde{M}_{\epsilon}\right)=1$. This completes the chain of implications and the theorem follows.

The next theorem is a generalization to $L^{\infty}$ functions of a result of Tanaka [6, Theorem 5], for $H^{\infty}$. By this time our proof is a considerable simplification of that given by Tanaka.

THEOREM 3.2. Let $f \in L^{\infty}$. Then, necessary and sufficient conditions for $f$ to be approximately continuous from above at 1 with value $\alpha$ are

(i) $f(h)=\alpha$ for all $h \in B^{+}$.

(ii) The set, $\left\{e^{i \theta}:\left|f\left(e^{i \theta}\right)\right| \leqq|\alpha|+\epsilon\right\}$, has density 1 at 1 from above for every $\epsilon>0$.

Note. In Tanaka's theorem condition (i) was the statement that $f$ tends to $\alpha$ radially. From Lemma 2.2 we see that this is equivalent to our (i) for $H^{\infty}$ functions.

Proof. First suppose $f$ is approximately continuous from above at 1 with value $\alpha$. By Theorem 3.1, $f=\alpha$ on the support of the representing measure for each $h \in B^{+}$. Immediately, $f=\alpha$ on $B^{+}$. Condition (ii) is necessary because

$$
\left\{e^{i \theta}:\left|f\left(e^{i \theta}\right)-\alpha\right| \leqq \epsilon\right\} \subset\left\{e^{i \theta}:\left|f\left(e^{i \theta}\right)\right| \leqq|\alpha|+\epsilon\right\} .
$$

Next, suppose the conditions (i) and (ii) are satisfied. Let $N_{\text {c }}$ $=\left\{e^{i \theta}:\left|f\left(e^{i \theta}\right)\right| \leqq|\alpha|+\epsilon\right\}$. By Corollary 2.6 and condition (ii), $\tilde{N}_{\epsilon}$ contains the support of the representing measure of each upper barely tangential homomorphism for every $\epsilon>0$. Thus, for every $\epsilon>0$ we have $|f| \leqq|\alpha|+\epsilon$ on each such support. Thus, $|f| \leqq|\alpha|$ on each such support. By condition (i) if $h \in B^{+}$, then $f(h)=\alpha$. But, $f(h)$ is the integral average of values not exceeding $\alpha$. Therefore, $f$ must be identically $\alpha$ on the support of $\mu_{h}$. By Theorem 3.1, again, $f$ is approximately continuous from above at 1 with value $\alpha$.

That condition (i) is necessary is a Lindelöf-type theorem for $L^{\infty}$. Using Lemma 2.3 we state this theorem more concretely. It should be noted that because of Lemma 2.2 this theorem generalizes the usual Lindelöf theorem for $H^{\infty}$ : If $f \in H^{\infty}$ and is approximately continuous from above at 1 with value $\alpha$, then $f$ tends to $\alpha$ radially.

TheOREM 3.3. Let $f \in L^{\infty}$. If $f$ is approximately continuous from above at 1 with value $\alpha$, then the upper barely tangential cluster set of $f$ at 1 consists of the single point $\alpha$. 


\section{REFERENCES}

1. T. Boehme, M. Rosenfeld and M. L. Weiss, Relations between bounded analytic functions and their boundary functions, J. London Math. Soc. (2) 1 (1969), 609-618.

2. L. Carleson, Interpolations by bounded analytic functions and the corona problem, Ann. of Math. (2) 76 (1962), 547-559. MR 25 \#5186.

3. K. Hoffman, Banach spaces of analytic functions, Prentice-Hall Series in Modern Analysis, Prentice-Hall, Englewood Cliffs, N.J., 1962. MR 24 \#A2844.

4. - Bounded analytic functions and Gleason parts, Ann. of Math. (2) 86 (1967), 74-111. MR 35 \#5945.

5. M. Rosenfeld and M. L. Weiss, $A$ function algebra approach to a theorem of Lindelöf, J. London Math. Soc. (2) 2 (1970).

6. C. Tanaka, On the metric cluster values of the bounded regular function in the unit disk, Mem. School Sci. Engrg., Waseda Univ., Tokyo, No. 31 (1967), 119-129.

University of California, Santa Barbara, California 93106 